\title{
Quantum operation, quantum Fourier transform and semi-definite programming
}

\author{
Runyao Duan $^{\mathrm{a}, *, 1}$, Zhengfeng Ji ${ }^{\mathrm{a}, 1}$ Yuan Feng ${ }^{\mathrm{a}, 1}$, \\ Mingsheng Ying a,1 \\ ${ }^{a}$ State Key Laboratory of Intelligent Technology and Systems, Department of \\ Computer Science and Technology Tsinghua University, Beijing, China, 100084
}

\begin{abstract}
We analyze a class of quantum operations based on a geometrical representation of $d$-level quantum system (or qudit for short). A sufficient and necessary condition of complete positivity, expressed in terms of the quantum Fourier transform, is found for this class of operations. A more general class of operations on qudits is also considered and its completely positive condition is reduced to the well-known semi-definite programming problem.
\end{abstract}

Key words: Quantum operation, Complete positivity, Quantum Fourier transform, Semi-definite programming

PACS: 3.67.Lx, 3.67.-a

\section{Introduction}

Almost all quantum systems in the real world are open in the sense that they suffer from unwanted interactions with the outside world. The dynamics of an open quantum system is usually much more complicated than that of a closed one. One of the most important mathematical tools describing the dynamics of an open quantum system is quantum operation, which has been

\footnotetext{
* Corresponding author.

Email addresses: dry02@mails.tsinghua.edu.cn (Runyao Duan), jizhengfeng98@mails.tsinghua.edu.cn (Zhengfeng Ji), fengy99g@mails, tsinghua.edu.cn (Yuan Feng), yingmsh@mail.tsinghua.edu.cn (Mingsheng Ying).
}

1 This work was partly supported by the National Foundation of Natural Sciences of China (Grant No: 60273003). 
systematically studied after Kraus's seminal work [1]. Comparing with other mathematical formalisms coping with open quantum systems, such as master equation, Langevin equation and stochastic differential equation, quantum operations are especially appropriate for depicting discrete state change in discrete time. Recently, the rapid development of quantum information processing technology revives a wide interest on quantum operations due to the fact that quantum information processing systems, for example, quantum computers, suffer from outside noises inevitablly, and that mainly a discrete-time evolution is concerned in these systems. Indeed, quantum operation plays an important role in many active fields such as quantum computation, quantum information, quantum error-correcting codes and quantum fault-tolerant computation $[2,3,4]$ (for an excellent exposition, see [5] Chapter 8).

There are several equivalent ways to introduce the notion of quantum operation; one of which is given in terms of completely positive (often abbreviated as $\mathrm{CP}$ ) mappings. Let $\mathcal{H}$ denote the Hilbert space of the principal quantum system. A linear mapping $\kappa$ on $\mathcal{H}$ is positive if it always sends a positive operator to another positive one; and $\kappa$ is completely positive if, furthermore, the mapping $I \otimes \kappa$ is also positive where $I$ is the identity mapping acting on an arbitrary ancillary system. Complete positivity is a natural requirement of the real physical world without which the state of the composite system may be invalid after operations on its subsystem. Thus, a quantum operation is defined as a completely positive and trace-preserving linear mapping on the state space $\mathcal{H}$. Some representations of the CP mapping have been presented in [1], also in[6], and an important one is the operator sum representation:

$$
\mathcal{E}(\rho)=\sum_{k=1}^{N} A_{k} \rho A_{k}^{\dagger}
$$

where $A_{k}^{\dagger}$ is the conjugate transpose of $A_{k}$. To guarantee the trace-preserving property, the following completeness condition

$$
\sum_{k=1}^{N} A_{k}^{\dagger} A_{k}=I
$$

is also required. For some other descriptions of quantum operations, we refer to [5]. As trace-preserving quantum operation is a fundamental mathematical description of quantum channel, a thorough study on quantum operation will help us to understand the limits or capabilities of quantum information processing.

The main goal of this paper is to find the necessary and sufficient conditions for certain kinds of linear mappings to be quantum operations. To motivate our problem, let us consider the trace-preserving quantum operation $\kappa$ on a two-level quantum system (or qubit for short). We employ the very useful 
geometric tool of Bloch sphere to represent a qubit. Then every qubit can be depicted as a vector in it. Recall that the general form of the affine mapping on the Bloch sphere is

$$
\vec{r}=\left(r_{x}, r_{y}, r_{z}\right) \mapsto \vec{r}^{\prime}=M\left(r_{x}, r_{y}, r_{z}\right)+\left(c_{x}, c_{y}, c_{z}\right),
$$

where $\vec{r}$ and $\vec{r}^{\prime}$ are respectively the original and the image vectors under the mapping, $M$ is a $3 \times 3$ real matrix and $\vec{c}=\left(c_{x}, c_{y}, c_{z}\right)$ the displacement vector. It is easy to see that the effect of a quantum operation on qubits is just an affine mapping on the corresponding vectors. Conversely, a question naturally arises: whether or not every affine mapping on the Bloch sphere has a corresponding quantum operation? The answer to this question is unfortunately no. In fact, there are very simple affine maps that cannot be images of quantum operations $[7,8]$. A sufficient and necessary condition of the special case without any displacement has been found by several research groups $[7,9,10]$ and some special cases of $\left(c_{x}, c_{y}, c_{z}\right)$ are also considered. In [11], M. B. Ruskai, S. Szarek and E. Werner have completely solved the general problem for the qubit case. More precisely, given the transition matrix $M$ and displacement $\vec{c}$, the sufficient and necessary condition of when such a mapping is completely positive was presented there.

A qubit is the simplest quantum system which has been studied thoroughly. However, quantum systems of higher dimension also concern us in quantum information processing and are relatively less studied. This observation leads us to consider higher dimensional generalization of the above question. To the authors' best knowledge, the higher dimensional version of this question is still open. In the present paper, we will carefully examine the affine mappings in higher dimensional quantum systems, and give a partial answer to this question. This will certainly give some new insights to the field of quantum information processing and bring a useful mathematical tool for the solutions of some problems in this field.

The paper is organized as follows. In Section 2, we extend the vector representation from qubit to qudit case. This enables us to give a $d$-dimensional generalization of affine mappings. Some simple properties of such geometric representation are presented. In Section 3, we focus on a special class of higher dimensional affine mappings where the transition matrix is diagonalized and the displacement vector vanishes. A sufficient and necessary condition of when an mapping in this class is a quantum operation is found. With this condition, one can easily check whether a given mapping in the special case is an image of a quantum operation or not. Some well known results, for example, the universal NOT gate with optimal fidelity in the high dimensional state space, can be recovered. A more general case involving multiple qudits is also discussed. The most interesting thing is that this condition is deeply related to the quantum Fourier transform (QFT for short), and such a surprising connection is 
not easy to observe if we only deal with the case of qubits. In Section 4, we further consider higher dimensional affine mappings with diagonal transition matrix in which the displacement vector presents. They are of the most common affine mappings in practical use. The problem of determining when such an affine mapping is a quantum operation is then reduced to the semi-positive definite programming problem. We argue that this problem has no simple solution in the general case. However, we are still able to give a simple sufficient condition of it. Finally, we draw a brief conclusion in Section 5.

\section{$2 \quad$ A geometric representation of qudits}

It is well known that for qubit system, a wonderful geometric representation using Bloch sphere exists. To be specific, the density matrix $\rho$ of a qubit can be written as

$$
\rho=\frac{I+\vec{r} \cdot \vec{\sigma}}{2}
$$

where $\vec{r}=\left(r_{x}, r_{y}, r_{z}\right)$ is a 3-dimensional real vector with Euclidean norm $\|\vec{r}\| \leq$ 1. $\vec{\sigma}=(X, Y, Z)$ is a formal vector of Pauli matrices and $\vec{r} \cdot \vec{\sigma}=r_{x} X+r_{y} Y+r_{z} Z$ where $X, Y, Z$ are Pauli matrices,

$$
X=\left(\begin{array}{ll}
0 & 1 \\
1 & 0
\end{array}\right), Y=\left(\begin{array}{cc}
0 & -i \\
i & 0
\end{array}\right), Z=\left(\begin{array}{cc}
1 & 0 \\
0 & -1
\end{array}\right) \text {. }
$$

It is notable that $\|\vec{r}\|=1$ if and only if $\rho$ is a pure state, and $\rho$ is a mixed state whenever $\|\vec{r}\|<1$. Given a vector $\vec{r}$, we can completely determine the corresponding quantum state $\rho$ and vise versa. Furthermore, the dynamics of $\rho$ can be visualized through the affine operation on $\vec{r}$. The key of a qubit possessing the Bloch sphere vector representation is there exist a set of matrices $\{X, Y, Z\}$ which, together with the identity matrix $I$, forms a basis of the $2 \times 2$ matrix space.

To extend such a representation to a higher dimensional state space, we choose a set of generalized Pauli matrices [2] as the basis. Suppose we have an orthonormal basis $\{|k\rangle\}$ of a qudit space $\mathcal{H}$, if we define $X$ as $X|k\rangle=|k+1\rangle$, where the addition is modulo $d$, and $Z$ as $Z|k\rangle=\omega^{k}|k\rangle$, where $\omega=\exp \left(-\frac{2 \pi i}{d}\right)$ is the $d$ th unity root, then the whole set of the general Pauli matrices in qudit system can be expressed as $\left\{\sigma_{p, q}=X^{p} Z^{q}: p, q=0, \cdots, d-1\right\}$. For a detailed presentation of the properties the generalized Pauli matrices enjoy, we refer to [2]. Here we only list some of them related to our results (for simplicity, we define $\left.X_{i}=X^{i}, Z_{i}=Z^{i}\right)$ : 
(1) (Multiplication relations)

$$
\sigma_{i, j} \sigma_{i^{\prime}, j^{\prime}}=\omega^{i^{\prime} j} \sigma_{\left(i+i^{\prime}\right),\left(j+j^{\prime}\right)} .
$$

(2) (Hermitian relations)

(3) (Commutation relations)

$$
\begin{gathered}
X_{i}^{\dagger}=X_{d-i}, Z_{i}^{\dagger}=Z_{d-i}, \\
\sigma_{i, j}^{\dagger}=\omega^{i j} \sigma_{(d-i),(d-j)} .
\end{gathered}
$$

$$
\sigma_{i, j} \sigma_{i^{\prime}, j^{\prime}}=\omega^{\left(i^{\prime} j-i j^{\prime}\right)} \sigma_{i^{\prime}, j^{\prime}} \sigma_{i, j} .
$$

(4) (Orthogonal relations) Suppose the inner product of $d \times d$ linear space is defined as $(A, B)=\operatorname{tr}\left(A^{\dagger} B\right)$, then

$$
\left(\sigma_{i, j}, \sigma_{i^{\prime}, j^{\prime}}\right)=d \delta_{i, i^{\prime}} \delta_{j, j^{\prime}},
$$

where $\delta$ is the Kronecker function.

$$
\delta_{i, j}=\left\{\begin{array}{l}
0, \text { if } \quad i \neq j \\
1, \text { if } \quad i=j
\end{array} .\right.
$$

(5) The Pauli matrices $\left\{\sigma_{i, j}: i, j=0,1, \cdots, d-1\right\}$ form an orthogonal basis of $d \times d$ linear space $Q$. That is, for any $A \in Q$,

$$
A=\frac{\sum_{i, j} \operatorname{tr}\left(\sigma_{i j}^{\dagger} A\right) \cdot \sigma_{i, j}}{d} .
$$

The next proposition shows that on a higher dimensional space the matrix composed of all eigenvectors of $X$ corresponds exactly to the Fourier transform $F$.

Proposition 1 Suppose $F$ is the quantum Fourier transform on d-dimensional Hilbert state space $\mathcal{H}$, that is

$$
F|j\rangle=\frac{1}{\sqrt{d}} \sum_{k=0}^{d-1} e^{2 \pi i j k / d}|k\rangle,
$$

then $X_{k}=F^{\dagger} Z_{k} F$, where $\{|j\rangle: j=0,1,2, \cdots, d-1\}$ is an orthonormal basis of $\mathcal{H}$.

Proof. Simple, details omitted.

Using this set of generalized Pauli matrices in high dimensional space, we can decompose a density matrix $\rho$ of a qudit in the principal Hilbert space $\mathcal{H}$ as follows:

$$
\rho=\frac{\vec{r} \cdot \vec{\sigma}}{d}
$$


where $\vec{r}=\left(\operatorname{tr}\left(\sigma_{00}^{\dagger} \rho\right), \operatorname{tr}\left(\sigma_{01}^{\dagger} \rho\right), \cdots, \operatorname{tr}\left(\sigma_{d-1, d-1}^{\dagger} \rho\right)\right)$ is a $d^{2}$-dimensional vector and $\vec{\sigma}$ is the formal vector such that $\vec{\sigma}=\left(\sigma_{0,0}, \sigma_{0,1}, \cdots, \sigma_{d-1, d-1}\right)$, the formal inner product is then defined as

$$
\vec{r} \cdot \vec{\sigma}=\sum_{p, q=0}^{d-1} r_{p, q} \sigma_{p, q}
$$

The representation here is compatible with the Bloch vector notation since we can simply add $I$ and 1 to the Pauli matrices $\vec{\sigma}$ and $\vec{r}$ respectively. Moreover, some properties of Bloch vector notation preserve in the new representation. For example, $\|\vec{r}\|=\sqrt{d}$ when $\rho$ is a pure state; $\|\vec{r}\|<\sqrt{d}$ when $\rho$ is a mixed state, while $\|\vec{r}\|=1$ if and only if $\rho$ is the maximally mixed state $I / d$. It is also useful to point out that to make $\rho$ a legal quantum state, we always have $r_{0,0}=\operatorname{tr}\left(\sigma_{0,0}^{\dagger} \rho\right)=\operatorname{tr}(\rho)=1$.

Suppose quantum operation $\mathcal{E}$ on $\mathcal{H}$ maps $\rho$ to $\rho^{\prime}$ with the representation

$$
\rho^{\prime}=\frac{\vec{r}^{\prime} \cdot \vec{\sigma}}{d}
$$

a simple computation tells us there exists a $d^{2} \times d^{2}$ dimensional matrix $M$ such that

$$
\vec{r}^{\prime}=M \vec{r}
$$

In other words, every quantum operation leads to an affine mapping with the form

$$
\vec{r} \mapsto M \vec{r}
$$

Then the question raised in the introduction can be more explicitly stated as follows: is every affine mapping with the form (5) a quantum operation on state space $\mathcal{H}$ ? In general, the answer to this question is certainly no. Our purpose of this paper is then to characterize the affine mapping that corresponds to some quantum operation.

Before we go deep into this question, let us first examine some properties that transition matrix $M$ and vector $\vec{r}$ must satisfy. We say a quantum operation $\mathcal{E}$ is unital if $\mathcal{E}(I)=I$. Otherwise we call it is non-unital. Suppose transition matrix $M$ corresponds to a trace-preserving quantum operation $\mathcal{E}$, then $M$ has the block form

$$
M=\left(\begin{array}{cc}
1 & 0 \\
c & M^{\prime}
\end{array}\right),
$$

where $c$ is a $d^{2}-1$ dimensional vector(we often call it as displacement vector), $M^{\prime}$ is a $\left(d^{2}-1\right) \times\left(d^{2}-1\right)$ matrix. Furthermore, if $\mathcal{E}$ is unital, we have $c=0$ (vector). For $\rho$ is a positive operator with trace one, we get $\vec{r} \cdot \vec{\sigma}=(\vec{r} \cdot \vec{\sigma})^{\dagger}$ and 
$r_{00}=1$, or equivalently,

$$
r_{p, q}^{*}=\omega^{-p q} r_{-p,-q}, r_{00}=1
$$

where all the arithmetical operations of the indices are modulo $d$, so $r_{-p,-q}=$ $r_{d-p, d-q}$. The above equations can be considered as a general constraints on the vector $\vec{r}$. With this condition, we can use $d^{2}-1$ real numbers to represent a qudit state $\rho$.

Denote the set of vectors satisfying the above equations as $V_{d^{2}}$. A simple calculation shows that $V_{d^{2}}$ is close under addition and multiplication by a real number; that is, $\overrightarrow{r_{1}}+\overrightarrow{r_{2}} \in V_{d^{2}}$ and $t \overrightarrow{r_{1}} \in V_{d^{2}}$ provided that $\overrightarrow{r_{1}}, \overrightarrow{r_{2}} \in V_{d^{2}}$ and $t$ is a real number. Notice that when $t$ is a complex number, $t \overrightarrow{r_{1}} \in V_{d^{2}}$ does not necessarily hold. Suppose A is a $d^{2} \times d^{2}$ matrix which is a linear mapping on $V_{d^{2}}$, then $A \vec{r} \in V_{d^{2}}$ for all $\vec{r} \in V_{d^{2}}$ if and only if $(A \vec{r} \cdot \vec{\sigma})^{\dagger}=A \vec{r} \cdot \vec{\sigma}$ for any $\vec{r} \in V_{d^{2}}$. Let us further consider a special but very important case where $A$ is a diagonal matrix

$$
A=\operatorname{diag}\left(\lambda_{0,0}, \lambda_{0,1}, \cdots, \lambda_{d-1, d-1}\right)
$$

or simply we write $A=\operatorname{diag}(\vec{\lambda})$. Then the condition turns out to be $\lambda_{-p,-q}=$ $\lambda_{p, q}^{*}$. So if a quantum operation maps $\rho$ with the Bloch vector $\vec{r}$ to $\mathcal{E}(\rho)$ with the Bloch vector $M \vec{r}$, where

$$
M=\operatorname{diag}\left(\lambda_{0,0}, \lambda_{0,1}, \cdots, \lambda_{d-1, d-1}\right)=\operatorname{diag}(\vec{\lambda})
$$

is diagonal, then the following conditions must be satisfied:

$$
r_{p, q}=\omega^{p q} r_{-p,-q}^{*}, \lambda_{-p,-q}=\lambda_{p, q}^{*}
$$

For the case of qubits, i.e., $d=2$, we have the pauli matrices

$$
\sigma_{0,0}=I, \sigma_{0,1}=Z, \sigma_{1,0}=X, \sigma_{1,1}=X Z
$$

and $\omega=-1$, then the above conditions about $\vec{r}$ may be rewritten as

$$
r_{0,0}=1, r_{0,1}=r_{0,1}^{*}, r_{1,0}=r_{1,0}^{*}, r_{1,1}=-r_{1,1}^{*}
$$

that is, $r_{0,0}=1, r_{0,1}, r_{1,0}$ are real numbers and $r_{1,1}$ is pure imaginary number. The conditions about $M$ can be rewritten as

$$
\lambda_{0,0}=1, \lambda_{0,1}=\lambda_{0,1}^{*}, \lambda_{1,0}=\lambda_{1,0}^{*}, \lambda_{1,1}=\lambda_{1,1}^{*}
$$

all the four numbers $r_{0,0}, r_{0,1}, r_{1,0}$ and $r_{1,1}$ are real. Moreover, by introducing $Y=i X Z$, we can also make $r_{1,1}$ a real number. Then the qubit state $\rho$ and real vector $\vec{r}=\left(r_{0,0}, r_{0,1}, r_{1,0}, r_{1,1}\right)$ are one to one corresponding to each other when $|\vec{r}| \leq 2$, and the scalars of the four axes are all real numbers. 


\section{Affine mappings with diagonal transition matrix}

In this section, we consider the special affine mappings with diagonal transition matrices $M$ and no displacement vectors. The effect of these mappings is just a scalar multiplication by $\lambda_{p, q}$ in the direction $\sigma_{p, q}$, or

$$
\vec{r} \mapsto M \vec{r},
$$

where $M=\operatorname{diag}(\vec{\lambda})$ is a diagonal matrix. we can also write

$$
\mathcal{E}\left(\sigma_{p, q}\right)=\lambda_{p, q} \sigma_{p, q}, \quad p, q=0, \cdots, d-1
$$

It is well known that when qubits are considered, with a suitably chosen axis, many interesting quantum operations have this simple form in the Bloch sphere. The typical examples are bit flip channel and phase damping [5].

This section is devoted to find the necessary and sufficient condition of such affine mapping to be a quantum operation's image. As mentioned before, a key feature of a quantum operation is its complete positivity. To determine the complete positivity of $\mathcal{E}$, we need the following theorem attributed to Kraus $[1]:$

Theorem 1 Suppose $\mathcal{E}$ is a linear operation on Hilbert space $\mathcal{H}, \mathcal{R}$ is an ancillary Hilbert space with the same dimensionality with $\mathcal{H}$. Let $\{|k\rangle\}$ be an orthonormal basis for $\mathcal{H}$ and $\mathcal{R}$. Then $\mathcal{E}$ is $C P$ mapping if and only if the operator

$$
(I \otimes \mathcal{E})(|\alpha\rangle\langle\alpha|)
$$

is positive, where

$$
|\alpha\rangle=\sum_{k}|k\rangle|k\rangle / \sqrt{d}
$$

is the maximal entangled state of the composite system.

Using Kraus's theorem, to decide whether a given linear mapping is a CP one, we need only examine the positivity of eigenvalues of the operator $(I \otimes$ $\mathcal{E})(|\alpha\rangle\langle\alpha|)$.

We now are able to present one of the main results of this paper, namely, a CP condition for an affine mapping $\mathcal{E}$ with a diagonal transition matrix and no displacement vector. To one's surprise, this condition is essentially connected to the quantum Fourier transform $F$, which, as we all known, plays a crucial role in quantum computation (for example, quantum Fourier transform is a key step for the Shor's Factoring algorithm and Discrete Logarithm Problem[4]). To be specific, we have the following theorem. 
Theorem 2 The linear mapping defined in (6) is a CP one if and only if

$$
\left(F \otimes F^{\dagger}\right) \vec{\lambda} \geq 0 .
$$

where $\vec{\lambda}=\left(\lambda_{0,0}, \lambda_{0,1}, \cdots, \lambda_{d-1, d-1}\right)$.

Proof. Using the Kraus's theorem, we need to calculate $(I \otimes \mathcal{E})(|\alpha\rangle\langle\alpha|)$. So first we expand $|k\rangle\langle l|$ as the linear combinations of $\left\{\sigma_{i, j}\right\}$. By the orthogonality of $\sigma_{i, j}$, we get

$$
|k\rangle\langle l|=\sum_{m, n} \alpha_{m, n} \sigma_{m, n},
$$

where $\alpha_{m, n}=\operatorname{tr}\left(\sigma_{m, n}^{\dagger}|k\rangle\langle l|\right) / d=\omega^{-n l} \delta_{m+l, k} / d$. So

$$
\begin{aligned}
& (I \otimes \mathcal{E})(|\alpha\rangle\langle\alpha|) \\
= & \sum_{k, l}|k\rangle\langle l| \mathcal{E}(|k\rangle\langle l|) / d \\
= & \sum_{l, m, n, q} \omega^{-(q+n) l} \sigma_{m, q} \otimes \sigma_{m, n} \lambda_{m, n} / d^{3} \\
= & \sum_{m, n} \lambda_{m, n} \sigma_{m,-n} \otimes \sigma_{m, n} / d^{2} .
\end{aligned}
$$

The crucial point of the problem is that the set

$$
\left\{\sigma_{m,-n} \otimes \sigma_{m, n}\right\}
$$

form an Abelian group of order $d^{2}$, which means that this set is closed under the operation of matrix multiplication, matrix inversion, and its elements commute with each other. We prove commutation relation as follows:

$$
\begin{aligned}
& \left(\sigma_{m,-n} \otimes \sigma_{m, n}\right)\left(\sigma_{p,-q} \otimes \sigma_{p, q}\right) \\
= & \left(\sigma_{m,-n} \sigma_{p,-q}\right) \otimes\left(\sigma_{m, n} \sigma_{p, q}\right) \\
= & \left(\omega^{-n p+q m} \sigma_{p,-q} \sigma_{m,-n}\right) \otimes\left(\omega^{n p-q m} \sigma_{p, q} \sigma_{m, n}\right) \\
= & \left(\sigma_{p,-q} \otimes \sigma_{p, q}\right)\left(\sigma_{m,-n} \otimes \sigma_{m, n}\right) .
\end{aligned}
$$

Thus, we can simultaneously diagonalize the set of matrices $\left\{\sigma_{p,-q} \otimes \sigma_{p, q}\right\}$ as

$$
\sigma_{m,-n} \otimes \sigma_{m, n}=U D(m, n) U^{\dagger},
$$


where $D(m, n)$ is a diagonal matrix, and $U$ a unitary matrix. It must be noted that the matrix $U$ can be chosen as the same for all the matrices in the set. Then

$$
(I \otimes \mathcal{E})(|\alpha\rangle\langle\alpha|)=\frac{1}{d^{2}} \sum_{m, n} U \lambda_{m, n} D(m, n) U^{\dagger} .
$$

The diagonal entries give the desired eigenvalues of $(I \otimes \mathcal{E})(|\alpha\rangle\langle\alpha|)$. Define

$$
\left|\Phi_{s, t}\right\rangle=\frac{1}{\sqrt{d}} \sum_{k}|k\rangle \sigma_{t, s}|k\rangle
$$

one can easily check

$$
\left(\sigma_{m,-n} \otimes \sigma_{m, n}\right)\left|\Phi_{s, t}\right\rangle=\omega^{n t-s m}\left|\Phi_{s, t}\right\rangle
$$

So by (7) we have

$$
(I \otimes \mathcal{E})(|\alpha\rangle\langle\alpha|)\left|\Phi_{s, t}\right\rangle=\mu_{s, t}\left|\Phi_{s, t}\right\rangle
$$

where

$$
\begin{aligned}
\vec{\mu}_{s t} & =1 / d^{2} \sum_{m, n} \omega^{n t-s m} \lambda_{m, n} \\
& =1 / d \sum_{m, n}(F)_{s, m}\left(F^{\dagger}\right)_{t, n} \lambda_{m, n} \\
& =1 / d\left[\left(F \otimes F^{\dagger}\right) \vec{\lambda}\right]_{s, t}
\end{aligned}
$$

or in matrix representation,

$$
\vec{\mu}=\frac{1}{d}\left(F \otimes F^{\dagger}\right) \vec{\lambda}
$$

For $(I \otimes \mathcal{E})(|\alpha\rangle\langle\alpha|)$ is a Hermitian matrix, it is positive if and only if all its eigenvalues are non-negative. This leads to the relation

$$
\left(F \otimes F^{\dagger}\right) \vec{\lambda} \geq 0
$$

With this we complete the proof of the theorem.

There are many ways to gain the result in the theorem and some of them are simpler. But the method given here is a coherent one in the sense that when the displacement vector does not vanish it still works.

Now we consider further the case where $N$ qudits are involved. Suppose the generalized Pauli matrices on $d$-dimensional Hilbert space $\mathcal{H}$ are $\left\{\sigma_{p, q}\right.$ : $p, q=0, \cdots, d-1\}$. Use this set Pauli matrices, we can obtain an orthogonal basis of operators acting on $\mathcal{H}^{\otimes N}$. For the sake of convenience, we denote $i=\left(i_{1}, i_{2}, \ldots, i_{N}\right)$ and $j=\left(j_{1}, j_{2}, \ldots, j_{N}\right)$, then we define the orthogonal basis 
as $\pi_{i, j}=\sigma_{i_{1}, j_{1}} \otimes \sigma_{i_{2}, j_{2}} \cdots, \otimes \sigma_{i_{N}, j_{N}}$, where $i_{1}, j_{1}, \cdots, i_{N}, j_{N}$ are range from 0 to $d-1$. Then the following theorem is a straightforward generalization of Theorem 2.

Theorem 3 Let $\mathcal{H}$ be a d-dimensional Hilbert space, and let $\mathcal{E}$ be a linear mapping on Hilbert space $\mathcal{H}^{\otimes N}$ such that

$$
\mathcal{E}\left(\pi_{i, j}\right)=\lambda_{i, j} \pi_{i, j}
$$

Then $\mathcal{E}$ is $C P$ if and only if

$$
\left(F \otimes F^{\dagger}\right)^{\otimes N} \vec{\lambda} \geq 0
$$

Proof. Same as the previous theorem. We omit the details here.

We should point out here that the quantum Fourier transform $F$ in the above theorem is defined on $\mathcal{H}$. There is another Fourier transformation $F^{\prime}$ which is defined on $N d$-dimensional state space $\mathcal{H}^{\otimes N} . F^{\prime}$ is not equal to $F^{\otimes N}$ in general. This enables us to obtain two different conditions. However, this two conditions are not related since they describe different types of affine mappings which are directly related to the choice of basis. One basis is general Pauli matrices and the other is tensor products of general Pauli matrices.

As a conclusion of this section, we show some simple applications of the above two theorems. First, let us come back to the case of qubits. Notice that in this case, quantum Fourier transform $F$ is just the Hadamard gate

$$
H=(X+Z) / \sqrt{2}=\frac{1}{\sqrt{2}}\left(\begin{array}{cc}
1 & 1 \\
1 & -1
\end{array}\right)
$$

For $H^{\dagger}=H$, the $\mathrm{CP}$ condition given in Theorem 3.3 can be rewritten in a more brief form:

$$
H^{\otimes 2 N} \vec{\lambda} \geq 0 .
$$

When only one qubit involves, the condition deduces to $H^{\otimes 2} \vec{\lambda}$, or more explicitly(noticing that $\lambda_{0,0}=1$ )

$$
\begin{aligned}
& 1+\lambda_{0,1}+\lambda_{1,0}+\lambda_{1,1} \geq 0, \\
& 1-\lambda_{0,1}+\lambda_{1,0}-\lambda_{1,1} \geq 0, \\
& 1+\lambda_{0,1}-\lambda_{1,0}-\lambda_{1,1} \geq 0, \\
& 1-\lambda_{0,1}-\lambda_{1,0}+\lambda_{1,1} \geq 0,
\end{aligned}
$$

which is similar as the equation (12) in [11].

Since $\left(F \otimes F^{\dagger}\right)^{\otimes N} \vec{\lambda}$ has an entry $\sum \lambda_{i, j} / d^{N}$, a necessary condition for $\mathcal{E}$ to be a CP mapping is that $\sum \lambda_{i, j} \geq 0$. An interesting special case here is when all 
entries in $\vec{\lambda}$ have some same real value $p$ except $\lambda_{0,0}=1$. This is in fact a generalized depolarizing channel. In this case, we have two different eigenvalues: $1+\left(d^{2}-1\right) p$ and $1-p$. The CP condition then becomes

$$
-1 /\left(d^{2}-1\right) \leq p \leq 1
$$

This result is well known and here it comes out to be a direct corollary of our above theorem.

What we would like to point out here is that when $c$ is negative, $-1 /\left(d^{2}-1\right)$ is the limit value we can achieve in this operation. It is also easy to see that this value decreases with $d$ increasing. This means that when dimensionality increases, the state space becomes more complicate, and the constraints between the different axes are more rigor.

Let us consider further the case where $p=-1 /\left(d^{2}-1\right)$. The pure state $\rho=$ $|\psi\rangle\langle\psi|$ will be mapped to $\mathcal{E}(\rho)=(d I-\rho) /\left(d^{2}-1\right)$. If we treat the operation as an unprecise universal NOT operation, then the fidelity will be

$$
\left\langle\psi^{\perp}|\mathcal{E}(\rho)| \psi^{\perp}\right\rangle=\frac{d}{d^{2}-1},
$$

which has been proved optimal in the case of $d=2$, see $[12,13]$.

Recall from Section 2 that we have the constraints $\lambda_{p, q}=\lambda_{-p,-q}^{*}$. So these operations are not completely independent in different axes, and there are conjugate axes such as $\sigma_{p, q}$ and $\sigma_{-p,-q}$. Thus, the scalars of a CP operation in this two directions must be conjugated with each other. In the qubit case, since $\sigma_{i, j}$ are all self-conjugated, the entries of $\vec{\lambda}$ are all real numbers, and the operation is independent for different axes.

\section{A more general class of affine mappings}

What concerns us in the previous section are affine mappings without displacement vectors. In this section, we consider a more general affine mapping of the form:

$$
\vec{r} \mapsto M \vec{r},
$$

where

$$
M=\operatorname{diag}(\vec{\lambda})+\left(\begin{array}{ll}
0 & 0 \\
c & 0
\end{array}\right) .
$$


We denote the first column of $\left(\begin{array}{ll}0 & 0 \\ c & 0\end{array}\right)$ as $\vec{c}$. Intuitively, we call $\vec{c}$ as displacement vector. Such kind of affine mappings correspond the most useful non-unital quantum operations in practice. The problem in which we are interested remains when such an affine mapping is an image of a quantum operation. The following theorem is the another main results in our paper which establishes a connection between this problem and the semi-positive definite programming.

Theorem 4 The linear mapping defined in (11) is a CP one if and only if

$$
I \otimes \vec{c} \cdot \vec{\sigma}+\sum_{p, q} \lambda_{p, q} \sigma_{p,-q} \otimes \sigma_{p, q} \geq 0
$$

Proof. From

$$
\mathcal{E}\left(\sigma_{0,0}\right)=\lambda_{0,0} \sigma_{0,0}+\vec{c} \cdot \vec{\sigma}
$$

we get

$$
\mathcal{E}\left(\sigma_{p, q}\right)=\lambda_{p, q} \sigma_{p, q}, \quad(p, q) \neq(0,0)
$$

and

$$
(I \otimes \mathcal{E})(|\alpha\rangle\langle\alpha|)=\frac{1}{d^{2}}\left[I \otimes \vec{c} \cdot \vec{\sigma}+\sum_{p, q} \lambda_{p, q} \sigma_{p,-q} \otimes \sigma_{p, q}\right] .
$$

Then by the Kraus's Theorem, the claim in the theorem holds, and we complete the proof.

Since the set of matrices $\left\{\sigma_{p,-q} \otimes \sigma_{p, q}\right\}$ can be simultaneously diagonalized, we can rewrite the condition in the above theorem as

$$
U^{\dagger}(I \otimes \vec{c} \cdot \vec{\sigma}) U+D \geq 0
$$

where $D=\operatorname{diag}\left(\left(F \otimes F^{\dagger}\right) \vec{\lambda}\right) / d$.

There are many basic problems in quantum information fields which can be reduced to the most general version of the semi-positive definite programming problem; for examples, quantum state discrimination [14,15], state estimation [16], and quantum pattern recognition [17]. The above theorem provides us with an additional example of such problems. Indeed, given $\vec{c}$. To keep $\mathcal{E}$ as a $\mathrm{CP}$ mapping, we need to determine the range of the diagonal matrix $D$. Then through $D$ we can immediately get the range of $\vec{\lambda}$, for

$$
\vec{\lambda}=d\left(F^{\dagger} \otimes F\right) \vec{D}
$$

where $\vec{D}$ denotes the vector formed by the diagonal entries of $D$. Furthermore, the problem of determining the range of a diagonal matrix $D$ to keep $A+D \geq 0$ for a Hermitian matrix $A$ is exactly a semi-positive definite programming problem. 
As is well known, there has not yet a general analytic solution for the problem of semi-positive definite programming. We then cannot get a sufficient and necessary condition in an explicit form under which the linear mappings (11) are completely positive for the most general case. Here we propose instead a sufficient condition:

Corollary 1 A sufficient condition for the linear mapping defined in (11) to be a CP one is

$$
\|\vec{c}\| \leq \mu_{\min }
$$

where

$$
\mu_{\min }=\min _{s, t}\left[\left(F \otimes F^{\dagger}\right) \vec{\lambda}\right]_{s, t} / d
$$

and

$$
\|\vec{c}\|=\sqrt{\sum_{p, q}\left|c_{p, q}\right|^{2}}
$$

is the norm of the displacement vector.

The intuitive meaning of the conclusion is that the affine mapping (11) will be an image of a quantum operation if the norm of the displacement vector $\vec{c}$ is small enough. Of course, here $\vec{c}$ should satisfy the equation $\vec{c} \cdot \vec{\sigma}=(\vec{c} \cdot \vec{\sigma})^{\dagger}$.

Proof. Let $\vec{c} \cdot \vec{\sigma}=V D_{c} V^{\dagger}$ be the spectral decomposition of $\vec{c} \cdot \vec{\sigma}$, where $D_{c}$ is a diagonal matrix and $\mathrm{V}$ is some unitary matrix. Since $\operatorname{tr}(\vec{c} \cdot \vec{\sigma})^{2}=\|\vec{c}\|^{2}$, we have $\lambda_{c} \leq\|\vec{c}\|$, where $\lambda_{c}$ is the maximal absolute value of eigenvalues of $\vec{c} \cdot \vec{\sigma}$. So when (12) holds, $\mu_{\min } I+D_{c} \geq 0$. Finally, we have

$$
\begin{aligned}
I \otimes \vec{c} \cdot \vec{\sigma}+U D U^{\dagger} & \geq \mu_{\min } I+I \otimes \vec{c} \cdot \vec{\sigma} \\
& =I \otimes V\left(\mu_{\min } I+D_{c}\right) V^{\dagger} \\
& \geq 0,
\end{aligned}
$$

where $V$ is unitary. This completes the proof.

The term $I \otimes \vec{c} \cdot \vec{\sigma}+U D U^{\dagger}$ is quite complicated, so one may hope to derive a simple expression of $\vec{c}$, as what was done in the last section, which can be used to characterize the positivity of the original term $I \otimes \vec{c} \cdot \vec{\sigma}+U D U^{\dagger}$. Unfortunately, this is impossible in general. The reason is that $U D U^{\dagger}$ and $I \otimes \vec{c} \cdot \vec{\sigma}$ do not commute with each other, but they are orthogonal to each other; in other words, we have

$$
\operatorname{tr}\left(U D U^{\dagger}(I \otimes \vec{c} \cdot \vec{\sigma})\right)=0
$$

This implies that we cannot diagonalize these two terms simultaneously. Otherwise, one of them will be led into a trivial case. Suppose that

$$
U D U^{\dagger}=I, \lambda_{p, q}=0,(p, q) \neq(0,0), \lambda_{0,0}=1 .
$$


To keep

$$
I \otimes(I+\vec{c} \cdot \vec{\sigma}) \geq 0,
$$

it only needs

$$
I+\vec{c} \cdot \vec{\sigma} \geq 0 .
$$

In other words, in this special case, the only condition that guarantees the lefthand side of the above inequality is positive is then not to move $\vec{c}$ out the unit sphere (of course, we need to require that $\vec{c}$ is a valid geometry representation of a quantum state).

\section{Conclusion}

In this paper, we use the set of generalized Pauli matrices as a tool to present a new geometric representation of a quantum state of qudits. This enables us to introduce affine mappings into the space of qudits. Then we find a sufficient and necessary condition for a special class of affine mappings. This condition is also generalized to the case of $N$ qudits. Using this result, we can easily decide whether a given mapping in the class we specified is a CP mapping or not. As an application, we derive the optimal fidelity of the universal NOT on $d$-dimensional state space:

$$
F\left(\rho^{\perp}, \rho\right)=\frac{d}{d^{2}-1} .
$$

Furthermore, we can easily deduce that when $d=2$, or in the qubit case, this fidelity is optimal with the value

$$
F\left(\rho^{\perp}, \rho\right)=\frac{2}{3} .
$$

The most interesting thing that we discover here is that the $\mathrm{CP}$ condition for this kind of affine mappings is closely related to the quantum Fourier transform.

We also consider some more general affine mappings with diagonal transition matrices and displacement vectors. We point out that the problem of deciding the complete positivity of these mapping is equivalent to the semi-positive definite programming problem. So, it is impossible to derive a general symbolic solution of this problem. However, we still get a sufficient condition which says that when the norm of the displacement vector is small enough, operation under consideration is $\mathrm{CP}$.

Acknowledgement: We thank anonymous referee for helpful comments and informing us of a valuable reference. 


\section{References}

[1] K. Kraus, State, Effects, and Operations, Lecture notes in Physics, Vol 190 (Springer, Berlin,1983)

[2] D. Gottesman, axXiv quant-ph/9802007

[3] A. R. Calderbank, E. M. Rains, P. W. Shor, Phys. Rev. A, 54, 1098(1996).

[4] P. W. Shor, Phys. Rev. A, 52, 2493(1995).

[5] M. A. Nielsen, I. L. Chuang, Quantum Computation and Quantum Information. (Cambridge university press, 2000).

[6] M.-D. Choi, Linear Algebr. Appl., 10, 285 (1975)

[7] A. Fujiwara and P. Algoet, Phys. Rev. A, 59, 3290(1999)

[8] A. Fujiwara; H. Nagaoka, IEEE Trans. Info. Theory 44:3(1998) 1071-1086

[9] Barbara M. Terhal, Isaac L. Chuang, David P. DiVincenzo, Markus Grassl, and John A. Smolin, Phys. Rev. A, 60, 881C885 (1999)

[10] C. King, M. B. Ruskai, IEEE Trans. Info. Theory, 47, 192-209 (2001)

[11] M. B. Ruskai, S. Szarek and E. Werner, An analysis of completely-positive trace-preserving maps on $\mathcal{M}_{2}$, Lin. Alg. and App., 347, (2002), 159

[12] V. Buzek, M. Hillery, and R. F. Werner, Phys. Rev. A, 60, R2626(1999)

[13] J. Fiurek, Phys. Rev. A, 64, 062310 (2001)

[14] A. Chefles, Contemp. Phys,. 41, 401 (2000)

[15] X. M. Sun, S. Y. Zhang, Y. Feng, and M. S. Ying, Phys. Rev. A, 65, 044306(2002)

[16] K. Vogel and H. Risken, Phys. Rev. A, 40, 2847 (1989).

[17] K. Audenaert and B. De Moor, Phys. Rev. A, 65, 030302(2002) 